\title{
A Genetic Algorithm-based Antenna Selection Approach for Large-but-Finite MIMO Networks
}

\author{
Behrooz Makki, Anatole Ide, Tommy Svensson, Thomas Eriksson, Mohamed-Slim Alouini, Fellow, IEEE
}

\begin{abstract}
We study the performance of antenna selectionbased multiple-input-multiple-output (MIMO) networks with large but finite number of transmit antennas and receivers. Considering the continuous and bursty communication scenarios with different users' data request probabilities, we develop an efficient antenna selection scheme using genetic algorithms (GA). As demonstrated, the proposed algorithm is generic in the sense that it can be used in the cases with different objective functions, precoding methods, levels of available channel state information and channel models. Our results show that the proposed GAbased algorithm reaches (almost) the same throughput as the exhaustive search-based optimal approach, with substantially less implementation complexity.
\end{abstract}

\section{INTRODUCTION}

To address the demands on the next generation of wireless networks, the main strategy persuaded recently is the network densification [1]-[4]. One of the promising techniques to densify the network is to use many transmit antennas and/or receive terminals. This approach is referred to as large multiple-input-multiple-output (MIMO) in the literature.

In general, the more antennas the transmitter and/or the receiver are equipped with, the better the data rate/link reliability. Thus, the trend is towards asymptotically high number of antennas. However, large MIMO implies challenges such as hardware impairments and signal processing complexity which may limit the number of antennas in practice. Thus, it is interesting to analyze MIMO networks in the presence of large but finite number of antennas. Particularly, antenna selection algorithms, e.g., [1]-[4], in which only a set of antennas are activated based on the channel quality, are appropriate schemes to utilize the diversity of large MIMO systems with high perantenna power and few radio-frequency (RF) chains.

Antenna selection/beamforming based on Tabu search and priority-based genetic algorithm (GA) is considered in [5] and [6], respectively. The symbol error probability [7], the outage probability [8] and the bit error probability [9] of the network are analyzed for different antenna selection algorithms. Moreover, [10]-[12] and [13] develop different selection schemes to maximize the capacity in spatially correlated and uncorrelated conditions, respectively. Finally, e.g., [1]-[4] have recently studied the problem in massive MIMO networks.

In this paper, we elaborate on the performance of largebut-finite MIMO networks utilizing antenna selection. The results are obtained for the cases with continuous and bursty

Behrooz Makki, Tommy Svensson and Thomas Eriksson are with Chalmers University of Technology, Email: \{behrooz.makki, tommy.svensson, thomase\}@chalmers.se. Anatole Ide is with ESIEE AMIENS, Amiens, France, Email: ide_a@esiee-amiens.fr. Mohamed-Slim Alouini is with the King Abdullah University of Science and Technology (KAUST), Email: slim.alouini@kaust.edu.sa

978-1-4799-5863-4/14/\$31.00 (C)2014 IEEE communication models of the users, where in each time slot the users may request for data transmission with different probabilities. The contribution of the paper is twofold. 1) We develop an efficient antenna selection scheme based on the GAs [6]. With the proposed GA-based approach, the appropriate transmit antennas are activated based on the instantaneous channel quality such that the system performance is optimized. As we show, the algorithm is generic in the sense that it can be efficiently applied with different amount of channel state information (CSI) at the transmitters/receivers, various channel/data communication models, objective functions as well as precoding schemes. This introduces the proposed algorithm as a powerful tool for performance comparison of different algorithms in a broad range of communication scenarios. 2) We compare the performance of our proposed approach with the state-of-the-art schemes and evaluate the effect of different parameters such as imperfect CSI, number of users/antennas and the users' data request probability on the performance of antenna selection-based MIMO setups. Particularly, for the simulations we consider the sum throughput and the Jain index [14] as two performance metrics in extreme cases with opportunistic and fair data transmission.

Our results, which are derived based on simulations, indicate that 1) the proposed GA-based antenna selection scheme reaches (almost) the same performance as in the optimal exhaustive search-based approach, with substantially less implementation complexity. Moreover, 2) the algorithm is effectively applicable for various convex and non-convex performance metrics. Also, 3) in the optimal case, the network Jain index is almost insensitive to the amount of CSI available at the transmitter, if zero-forcing precoder is implemented. The throughput, on the other hand, is significantly affected by the amount of CSI at the transmitter.

The problem setup of the paper is different from, e.g., [1]-[13], because we consider continuous and bursty data communication scenarios and concentrate on the cases with different channel/CSI models. Moreover, the proposed GAbased algorithm has not been presented before.

\section{SYSTEM MODEL}

Consider a multiuser MIMO setup with $M$ transmit antennas and a maximum of $\tilde{N} \leq M$ single-antenna users. With a bursty communication model, in each time slot different users may request for data transmission with probability $\alpha$. Note that setting $\alpha=1$ represents the continuous communication model where all users are always active requesting for data transmission. In this way, with $N$ data requesting users at time slot $t$, the received signal is given by

$$
\mathbf{y}(t)=P \mathbf{H}(t) \mathbf{V}(t) \mathbf{s}(t)+\mathbf{z}(t), \mathbf{z}(t) \in \mathcal{C}^{N \times 1} .
$$


Here, representing the number of selected transmit antennas by $m, \mathbf{H}(t) \in \mathcal{C}^{N \times m}$ is the fading matrix with the $(i, j)$ th element given by $H_{i, j}(t)=d_{i, j}^{\zeta_{i, j}} h_{i, j}(t)$ where $d_{i, j}$ is the distance between the receiver $i$ and antenna $j, \zeta_{i, j}$ is given by the path loss exponent and $h_{i, j}(t) \in \mathcal{C}$ denotes the small scale fading. Then, $\mathbf{s}(t)$ denotes the transmitted message, $\mathbf{V}(t)$ is the precodig matrix and $\mathbf{z}(t) \in \mathcal{C}^{N \times 1}$ denotes the independent and identically distributed (IID) complex Gaussian noise matrix with normalized variance. Also, $P$ represents the total power budget and, depending on the precoder type, different power normalization constraints can be considered for the precoder. To simplify the presentation, we drop the time index $t$ in the following.

The channel coefficients are assumed to be known by the receivers which is an acceptable assumption in block-fading conditions [2]-[4], [7]-[13]. However, this is not a necessary assumption and, using [15], the results can be extended to the cases with imperfect CSI at the receivers. On the other hand, the transmitter is provided with imperfect CSI modeled by

$$
\mathbf{H}=\beta \hat{\mathbf{H}}+\sqrt{1-\beta^{2}} \tilde{\mathbf{H}},
$$

where $\hat{\mathbf{H}}$ and $\tilde{\mathbf{H}}$ denote the known and the unknown parts of the channel, respectively. Also, $\beta \in[0,1]$ determines the quality of the CSI available at the transmitter with $\beta=0$ (resp. $\beta=1$ ) representing the cases with no (resp. perfect) CSI at the transmitter. In this way, the precoder is designed based on $\hat{\mathbf{H}}$ known by the transmitter.

As we show, the proposed algorithm can be used for a broad range of performance metrics. However, in the simulations, we concentrate on the sum throughput and Jain index, as objective functions in opportunistic and fair data transmission scenarios, respectively. These metrics are defined as follows.

Denote the set of data requesting users in a time slot by $\mathcal{X} \subseteq\{1, \ldots, \tilde{N}\}$, and the cardinality of $\mathcal{X}$ by $C_{\mathcal{X}}$. Then, the sum throughput, averaged over many time slots, is given by

$$
\eta=\sum_{\forall \mathcal{X}} \operatorname{Pr}(\mathcal{X}) E\{R(\mathbf{H} \mid \mathcal{X})\} \stackrel{(a)}{=} \sum_{N=1}^{\tilde{N}} \operatorname{Pr}(N) E\{R(N)\} .
$$

Here,

$$
\operatorname{Pr}(\mathcal{X})=\alpha^{C \mathcal{X}}(1-\alpha)^{\tilde{N}-C_{\mathcal{X}}}
$$

is the probability that specific users $n \in \mathcal{X}$ request for data transmission (and the rest remain silent). Also, the probability that $N$ users request for data transmission, independently of the users' indices, is given by

$$
\operatorname{Pr}(N)=\left(\begin{array}{c}
\tilde{N} \\
N
\end{array}\right) \alpha^{N}(1-\alpha)^{\tilde{N}-N},
$$

with $\left(\begin{array}{l}n \\ k\end{array}\right)$ being the " $n$ choose $k$ " operator. Thus, $(a)$ in (3) holds in the cases with identical long-term channel statistics of the users, on which we concentrate in the simulations. Then, denoting the expectation operator by $E\{\cdot\}, E\{R(\mathbf{H} \mid \mathcal{X})\}$ stands for the expected achievable throughput given the data requesting users $n \in \mathcal{X}$, with expectation over all possible channel realizations $\mathbf{H}$.

Assuming the cases with no interference cancellation at the users and $m$ transmitting antennas with equal average power allocation, the achievable rate terms $R(\mathbf{H} \mid \mathcal{X})$ and $R(N)$ are respectively obtained by

$$
R(\mathbf{H} \mid \mathcal{X})=\sum_{\forall i \in \mathcal{X}} \log _{2}\left(1+\frac{P g_{i, i}}{P \sum_{\forall j \in \mathcal{X}, j \neq i} g_{i, j}+1}\right),
$$

and

$$
R(N)=\sum_{i=1}^{N} \log _{2}\left(1+\frac{P g_{i, i}}{P \sum_{j=1, j \neq i}^{N} g_{i, j}+1}\right),
$$

bits per channel use (bpcu), where $g_{i, j}$ is the $(i, j)$-th element of the matrix $\mathbf{G}=|\mathbf{H V}|^{2}$.

Following the same procedure, the Jain index which rates the fairness between the users is defined as [14]

$$
\mathcal{J}=\sum_{N=1}^{\tilde{N}} \operatorname{Pr}(N) E\{J(N)\},
$$

$J(N) \doteq \frac{\left(\sum_{i=1}^{N} r_{i}\right)^{2}}{N \sum_{i=1}^{N} r_{i}^{2}}, r_{i} \doteq \log _{2}\left(1+\frac{P g_{i, i}}{P \sum_{j=1, j \neq i}^{N} g_{i, j}+1}\right)$

Note that with $N$ users the Jain index ranges between $\mathcal{J}=\frac{1}{N}$ for the least fair distribution to $\mathcal{J}=1$ for the fairest distribution. Therefore, to have a fair system the objective is to approach $\mathcal{J}=1$. Also, among our motivations for the Jain index analysis is to highlight the effectiveness of the proposed algorithm in optimizing the non-convex criteria.

\section{Algorithm Description}

With a total power budget, the per-antenna power may decrease by increasing the number of transmitting antennas. On the other hand, the MIMO diversity increases with the number of antennas. Thus, there is a tradeoff and, depending on the instantaneous channel condition, there may be an optimal set of antennas optimizing the system performance. Considering our general problem formulation/systm model, it is difficult to derive the optimal antenna selection schemes analytically. Also, with $M$ transmit antennas, there are $2^{M}$ possible antenna selection strategies. Therefore, the optimal set of antennas can indeed be selected via exhaustive search in the cases with few antennas. However, as the network size grows, which is of interest in the next generation of wireless networks, we need to design efficient algorithms to derive the (sub)optimal antenna selection with low complexity ${ }^{1}$.

In this paper, we propose a GA-based antenna selection approach as explained in Algorithm 1. In words, the algorithm is based on the following procedure. Start the algorithm by considering $K$ possible antenna selection strategies. Each strategy corresponds to a selected set of antennas, i.e., selecting a sub-matrix of matrix $\mathbf{H} \in \mathcal{C}^{N \times M}$. In each iteration, we determine the best strategy, referred to as the queen, that results in the best value of the considered utility function, compared to the other considered strategies (for instances, the highest throughput if the sum throughput is the objective function). Then, we keep the queen for the next iteration and create $J<K$ matrices around the queen. This is achieved by applying small modifications to the queen; for example, by changing a few number of antennas in the set of antennas

\footnotetext{
${ }^{1}$ For example, with $M=100$ the number of possible selections in the exhaustive search is of order $10^{30}$.
} 
associated with the queen or by adding/omitting a number of antennas in the queen. Also, in each iteration $K-J-1$ sets of antennas are selected randomly and the iterations continue for $N_{\text {it }}$ times considered by the designer. Running all considered iterations, the queen is returned as the antenna selection rule of the current network realization. The appropriate parameter setting for $K$ and $J$ in the algorithm can be found by simulations. Particularly, our simulations show that the proper values of $K$ and $J$ are almost insensitive to $\tilde{N}$ and $M$, and for different cases we have observed fairly good convergence speed by setting $J=\frac{K}{2}$.

\section{Algorithm 1 GA-based Antenna Selection Algorithm}

$\overline{\text { In each time slot with } N \text { data requesting users and the instan- }}$ taneous channel realization $\mathbf{H} \in \mathcal{C}^{N \times M}$ from all antennas to the data requesting users, do the followings:

I. Consider $K$, e.g., $K=10$, sets of antennas $\mathcal{M}_{k}, k=$ $1, \ldots, K$, and for each set create the corresponding subchannel matrix from $\hat{\mathbf{H}} \in \mathcal{C}^{N \times M}$ which is known by the transmitter (see (2)); consequently, $K$ associated matrices $\hat{\mathbf{H}}_{k} \in \mathcal{C}^{N \times m_{k}}, k=1 \ldots, K$, are created where $m_{k}$ is the cardinality of the set $\mathcal{M}_{k}$.

II. For each matrix $\hat{\mathbf{H}}_{k}, k=1 \ldots, K$, design the considered precoding scheme and evaluate the instaneous value of the objective function $U_{k}, k=1, \ldots, K$. For instance, considering the throughput (resp. the Jain index) as the objective function, the instaneous value of $U_{k}$ is given by (6) (resp. (9)).

III. Find the set of antennas which results in the best value of the objective function (the queen), e.g., $\mathcal{M}_{i}$ where $R\left(\hat{\mathbf{H}}_{k}\right) \leq R\left(\hat{\mathbf{H}}_{i}\right), \forall k=1, \ldots, K$, if the throughput is the objective function.

IV. $\mathcal{M}_{1} \leftarrow \mathcal{M}_{i}$.

V. Generate $J \ll K$, e.g., $J=5$, sets of antennas $\mathcal{M}_{j}^{\text {new }}, j=1, \ldots, J$, around $\mathcal{M}_{i}$. These sets are generated by small changes in the queen; for instance, by replacing few antennas with another set of antennas or by adding/omitting a number of antennas in the queen.

VI. $\mathcal{M}_{j+1} \leftarrow \mathcal{M}_{j}^{\text {new }}, j=1, \ldots, J$.

VII. Regenerate the remaining sets $\mathcal{M}_{j}, j=J+2, \ldots, K$, randomly with the same procedure as in Step I.

VIII. Go to Step II. and continue for $N_{\text {it }}$ iterations where $N_{\text {it }}$ is the number of iterations considered by the designer.

Return the queen as the antenna selection rule of the current time slot

Considering Algorithm 1, it is interesting to note that:

1) the algorithm is independent of the channel model and can be implemented in different data communication models. Also, the proposed algorithm is applicable for various objective functions/precoding schemes.

2) The GA-based algorithms are commonly considered as slow optimization schemes. However, as seen in the following, the proposed algorithm leads to (almost) the same results as the optimal (exhaustive-search) antenna selection with few number of iterations. Therefore, the algorithm is reasonably fast and it can be implemented for antenna selection when the number of antennas increases (see Section III.A for complexity analysis).

3) Due to step VII. of the algorithm, where $K-J-1$ random channel assignments are checked in each iteration, the proposed GA-based algorithm mimics the exhaustive search if $N_{\text {it }} \rightarrow \infty$ and it reaches the globally optimal selection rule if infinitely many iterations are considered. That is, the proposed scheme is optimal when the number of iterations increases asymptotically.

4) For generality, Algorithm 1 considers no constraint on the number of transmit antennas. However, Steps I, V and VII can be easily adapted such that the considered number of transmit antennas is not less than the number of users, and the multiplexing gain is guaranteed.

4) Finally, while we presented the algorithm for antenna selection in the cases with a single transmitter, it can be well applied in different complex optimization problems such as user scheduling in MIMO broadcast networks and resource allocation in return-link multi-beam satellite systems/distributed MIMO networks.

\section{A. On the Implementation Complexity}

The proposed algorithm leads to significantly less implementation complexity, compared to exhaustive search. This is because the algorithm implies $K N_{\text {it }}$ trials of antenna assignments which, depending on the considered parameter settings, can be considerably low (see Table I and Fig. 1 for example results). The proposed algorithm may not be the most lowcomplex algorithm in the literature; instead its advantage lies in its generality, in the sense that it can be implemented for different channel models, precoding schemes, available CSI, etc. Therefore, our proposed scheme can be considered as a yardstick for performance evaluation of different algorithms, and investigate the effect of different channel conditions/data transmission techniques on the network performance.

Finally, as an illustrative example, we derive the complexity in the cases with zero-forcing precoder, continuous communication and the sum throughput as the objective function. Here, due to symmetry, the considered matrices $\hat{\mathbf{H}}_{k}, k=1, \ldots, K$, are of size $N \times m_{k}$ with $m_{k} \in[1, M]$ and $\operatorname{Pr}\left(m_{k}\right)=\frac{1}{M}$. Then, considering the "pinv" function of MATLAB (with complexity $\mathcal{O}\left(\max \left(N, m_{k}\right) \min \left(N, m_{k}\right)^{2}+\right.$ $\left.\left.\min \left(N, m_{k}\right)^{3}\right)\right)$ and the products of two $N \times m_{k}$ matrices (with complexity $\mathcal{O}\left(N^{2} m_{k}\right)$ ) to calculate the precoding and the received signal-to-interference-plus-noise ratio (SINR) matrices, respectively, the algorithm complexity is given by $\frac{K N_{\text {it }}}{M} \sum_{m_{k}=1}^{M}\left\{\mathcal{O}\left(\max \left(N, m_{k}\right) \min \left(N, m_{k}\right)^{2}+\right.\right.$ $\left.\left.\min \left(N, m_{k}\right)^{3}\right)+\mathcal{O}\left(N^{2} m_{k}\right)\right\}$; This can be low complexity, depending on the parameters $K, N_{\text {it }}$. Also, the complexity decreases in the cases with bursty communication.

\section{Simulation RESUlts}

For the simulation results, we consider Rayleigh-fading conditions, use zero-forcing precoder with an average power normalization constraint and set $d_{i, j}=1, \forall i, j$. In the meantime, we have tested the algorithm for different large-scaling fading factors which show the same qualitative conclusions as in the presented figures. In all figures, we have considered 
$5 \times 10^{5}$ different channel realizations for each point in the simulation curves. Figures 1-2, 4 consider continuous data communication model $(\alpha=1)$. Then, with a bursty communication setup, we investigate the effect of the users' data request probability on the sum throughput in Fig. 3. In all figures, except Fig. 1, the algorithm is run for sufficiently large number of iterations until no further performance improvement is observed by increasing the number of iterations. Then, in Fig. 1 and Table I we study the performance of the proposed antenna selection approach for different numbers of iterations. Here, the results are presented for $K=10$ and $J=5$. In the meantime, we have checked the results for other parameter settings of the algorithm as well. Finally, the sum throughput is considered as the metric in Figs. 1-3, while Fig. 4 studies the network Jain index in a fair data transmission scenario.

Figure 1 shows an example for the convergence of the proposed GA-based algorithm in the cases with $M=100, \tilde{N}=$ $20, K=10, J=5, \beta=0.4, P=10 \mathrm{~dB}$. Here, we plot the relative achievable throughput $\Delta=\frac{\eta_{N_{\text {it }}}}{N_{\infty}} \%$, where $\eta_{N_{\text {it }}}$ is the throughput achieved with $N_{\text {it }}$ iterations. Also, $\eta_{\infty}$ denotes the maximum achievable throughput with asymptotically high number of iterations of the algorithm, which is the same as the throughput of the exhaustive search-based approach.

Setting $K=10$, Table I shows the average number of iterations that are required in the proposed algorithm to achieve the maximum exhaustive search-based throughput. Also, the table compares the performance of the proposed algorithm with $J=0,5$ and the cases where in each iteration all candidates, except the queen, are selected randomly, i.e., $J=9$. In parallel to our work, [5] has recently developed an algorithm based on Tabu search for beamforming in millimeter-wave communication. There, while the same conceptual procedure as in our work is followed to search around the solution candidates, specific criteria are set to generate neighbors around the queen, and Step VII of our algorithm which is used to reduce the effect of local minima is not considered. In Table I, we compare the performance of our scheme and the ones derived by considering the neighbor generation method of [5] in the cases with and without Step VII.

Considering continuous communication, Fig. 2 demonstrates the effect of the available CSI on the throughput. Here, we set $M=80, \tilde{N}=10$ and plot the relative throughput $\tilde{\Delta}=\frac{\eta(\beta)}{\eta(\beta=1)} \%$ where $\eta(\beta)$ is the throughput achieved with imperfect CSI modeled by parameter $\beta$ in (2). Also, $\eta(\beta=1)$ denotes the maximum achievable throughput with perfect CSI at the transmitter (see (2)). With different levels of imperfect CSI at the transmitter, Fig. 3 shows the network sum throughput for different users' data request probabilities in a bursty communication setup. Here, the results are presented for the cases with $M=40, \tilde{N}=20, \beta=0.4,0.8$, and $P=5,7 \mathrm{~dB}$.

Finally, Fig. 4 shows the performance of the proposed algorithm in a fair data transmission scenario with Jain index being the objective function. Here, the results are presented for $M=80, \tilde{N}=10$ and different levels of CSI at the transmitter. According to the figures, the following points are concluded:

- On the performance of the proposed scheduler: As seen in Fig. 1 and Table I, the developed scheduler leads to (almost) the same performance as the exhaustive search-

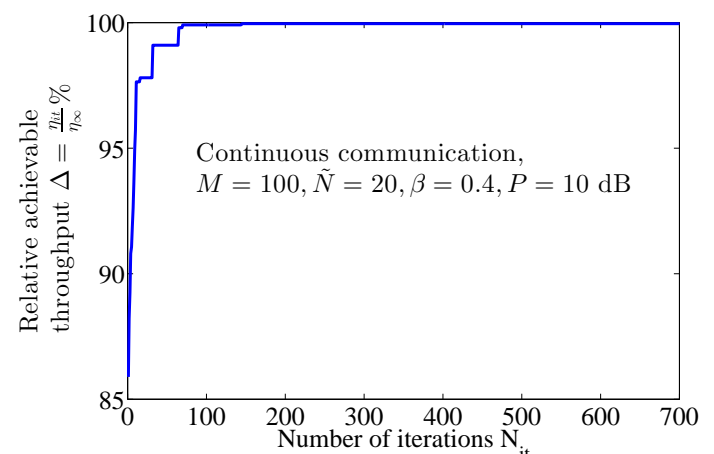

Figure 1. An example of the convergence process of the proposed algorithm. Continuous communication, $\tilde{N}=20, M=100, \beta=0.4, P=10 \mathrm{~dB}$. Table I

EXAMPLES FOR THE AVERAGE NUMBER OF ITERATIONS REQUIRED FOR THE CONVERGENCE OF THE ALGORITHM. THE RESULTS ARE AVERAGED OVER $5 \times 10^{4}$ DIFFERENT RANDOM CHANNEL REALIZATIONS.

\begin{tabular}{|c|c|c|c|}
\hline & $M=60$ & $M=70$ & $M=100$ \\
\hline GA, $J=5$ & 6373 & 6460 & 6602 \\
\hline GA, $J=0$ & $>10000$ & $>10000$ & $>10000$ \\
\hline GA, $J=9$ & 7437 & 7600 & 7691 \\
\hline Tabu without Step VII, $J=0$ & $>10000$ & $>10000$ & $>10000$ \\
\hline Tabu with Step VII, $J=5$ & 6919 & 6949 & 7580 \\
\hline
\end{tabular}

based scheduler with very limited number of iterations (note that with the parameter settings of the figure, exhaustive search implies testing in the order of $10^{30}$ possible antenna selection strategies). For instance, with the parameter settings of Fig. 1, the proposed algorithm reaches more than $98 \%$ of the maximum achievable throughput with less than 50 iterations (in all tested channel realizations we observed $95 \%$ of the maximum achievable throughput with less than 100 iterations). As seen, the algorithm converges in a ladder fashion. This is because the system performance is not necessarily improved in each iteration and it may reach a local optimum in some iterations. However, due to Steps V and VII of the algorithm, it can always escape a local minima and reach the global optimum if sufficiently large number of iterations are considered.

- Comparison of different schemes: Searching around the queen, i.e., Step V of Algorithm 1, can effectively improve the convergence speed and, compared to the cases with $J=9$, the required number of iterations reduces considerably if we set $J=5$ (compare Rows 1 and 3 of Table I). With and without neighbor generation

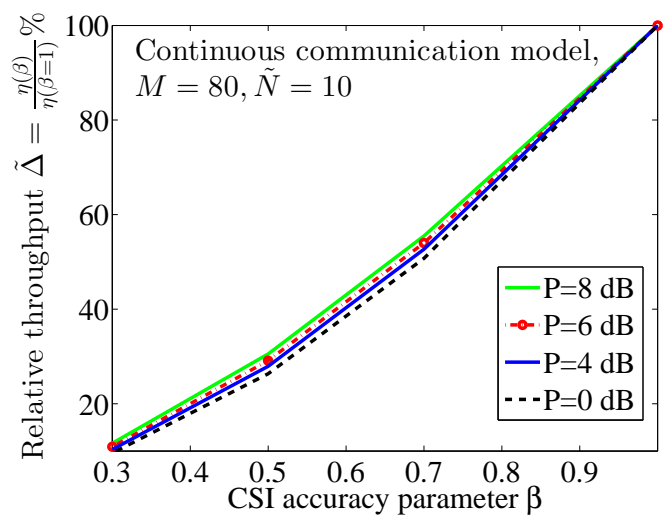

Figure 2. The relative throughput $\tilde{\Delta}=\frac{\eta(\beta)}{\eta(\beta=1)} \%$ for different imperfect CSI levels. Continuous communication, $\tilde{N}=10, M=80$. 
constraints of [5], ignoring Step VII of the algorithm increases the required number of iterations significantly (Table I, Rows 2 and 4). This is intuitively because, without Step VII of the algorithm, there is high probability that the algorithm is trapped in a local minima. Also, considering constraints on neighbor generation increases the required number of iterations, compared to the cases with no constraint on neighbor generation, intuitively because the constraints limit the possible solutions that can be checked in each iteration (compare Rows 1 and 5 of Table I). Finally, selecting the best algorithm is not easy since the decision depends on several parameters such as the matrices sizes, affordable complexity, problem formulation and the neighbor generation complexity.

- On the effect of imperfect CSI: In the optimal case, the network Jain index is (almost) not sensitive to the amount of CSI available at the transmitter (Fig. 4). On the other hand, the sum throughput is significantly affected by the CSI at the transmitter (Fig. 2). Also, with imperfect CSI, the relative system throughput is sensitive to the transmit power at moderate values of $\beta$, while its sensitivity decreases as $\beta$ increases.

- On bursty communication: With a bursty communication setup, the sum throughput is sensitive to small users' data request probabilities. However, the throughput sensitivity decreases as the users' data request probability, i.e., $\alpha$, increases. Moreover, the proposed algorithm is well applicable for different continuous and bursty data communication models.

- On fairness: The Jain index decreases (resp. increases) slightly with the transmit power (resp. available CSI). However, the changes are negligible and, with different parameter settings and zero-forcing precoder, the algorithm can be effectively utilized to guarantee fairness between the users (Fig. 4). This also emphasizes the effectiveness of the proposed algorithm in the optimization of non-convex metrics.

\section{CONCLUSion}

We studied the performance of large-but-finite MIMO networks using antenna selection. Considering different users' bursty data request probabilities, we developed a GA-based antenna selection approach and evaluated the effect of different parameters on the network throughput and Jain index. As illustrated, in the optimal case, the network Jain index (resp. throughput) is almost insensitive (significantly sensitive) to the amount of CSI available at the transmitter, if zero-forcing precoder is used. Finally, the proposed algorithm reaches the maximum achievable throughput with few iterations. Therefore, the algorithm can be practically implemented for antenna selection in MIMO networks. The mathematical analysis of the algorithm convergence is an interesting extension of the paper.

\section{REFERENCES}

[1] X. Gao, O. Edfors, F. Tufvesson, and E. G. Larsson, "Massive MIMO in real propagation environments: Do all antennas contribute equally?" IEEE Trans. Commun., vol. 63, no. 11, pp. 3917-3928, Nov. 2015.

[2] X. Gao, O. Edfors, J. Liu, and F. Tufvesson, "Antenna selection in measured massive MIMO channels using convex optimization," in IEEE Globecom'2013, Dec. 2013, pp. 129-134.

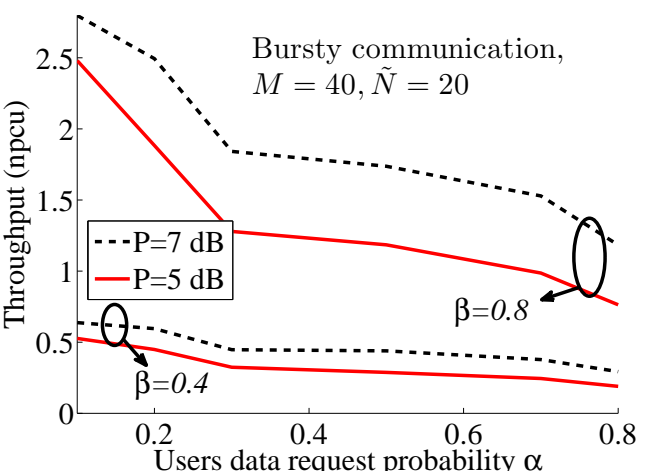

Figure 3. Throughput for different users' data request probabilities $\alpha, \tilde{N}=$ $20, M=50$.

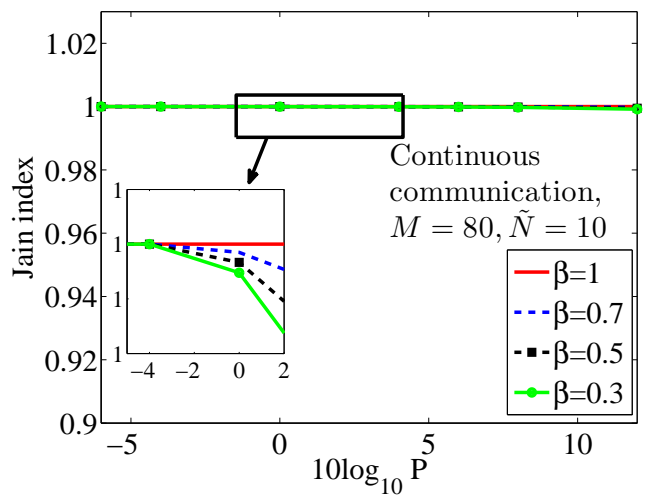

Figure 4. On the performance of the algorithm with Jain index considered as the objective function, $\tilde{N}=10, M=80$.

[3] X. Guozhen, L. An, J. Wei, X. Haige, and L. Wu, "Joint user scheduling and antenna selection in distributed massive MIMO systems with limited backhaul capacity," China Commun., vol. 11, no. 5, pp. 17-30, May 2014.

[4] B. Lee, L. Ngo, and B. Shim, "Antenna group selection based user scheduling for massive MIMO systems," in IEEE Globecom'2014, Dec. 2014, pp. 3302-3307.

[5] X. Gao, L. Dai, C. Yuen, and Z. Wang, "Turbo-like beamforming based on tabu search algorithm for millimeter-wave massive MIMO systems," IEEE Trans. Veh. Technol., vol. 65, no. 7, pp. 5731-5737, July 2016.

[6] H. Y. Lu and W. H. Fang, "Joint transmit/receive antenna selection in MIMO systems based on the priority-based genetic algorithm," IEEE Antennas Wireless Propag. Lett., vol. 6, pp. 588-591, 2007.

[7] D. A. Gore and A. J. Paulraj, "MIMO antenna subset selection with space-time coding," IEEE Trans. Signal Process., vol. 50, no. 10, pp. 2580-2588, Oct. 2002.

[8] H. Shen and A. Ghrayeb, "Analysis of the outage probability for MIMO systems with receive antenna selection," IEEE Trans. Veh. Technol., vol. 55, no. 4, pp. 1435-1440, July 2006.

[9] S.-H. Tsai, "Equal gain transmission with antenna selection in MIMO communications," IEEE Trans. Wireless Commun., vol. 10, no. 5, pp. 1470-1479, May 2011.

[10] L. Dai, S. Sfar, and K. B. Letaief, "Optimal antenna selection based on capacity maximization for MIMO systems in correlated channels," IEEE Trans. Commun., vol. 54, no. 3, pp. 563-573, March 2006.

[11] Z. Xu, S. Sfar, and R. S. Blum, "Analysis of MIMO systems with receive antenna selection in spatially correlated Rayleigh fading channels," IEEE Trans. Veh. Technol., vol. 58, no. 1, pp. 251-262, Jan. 2009.

[12] C. S. Park and K. B. Lee, "Statistical multimode transmit antenna selection for limited feedback MIMO systems," IEEE Trans. Wireless Commun., vol. 7, no. 11, pp. 4432-4438, Nov. 2008.

[13] S. Sanayei and A. Nosratinia, "Capacity of MIMO channels with antenna selection," IEEE Trans. Inf. Theory, vol. 53, no. 11, pp. 4356-4362, Nov. 2007.

[14] A. V. Babu and L. Jacob, "Fairness analysis of IEEE 802.11 multirate wireless LANs," IEEE Trans. Veh. Technol., vol. 56, no. 5, pp. 30733088, Sept. 2007.

[15] M. Medard, "The effect upon channel capacity in wireless communications of perfect and imperfect knowledge of the channel," IEEE Trans. Inf. Theory, vol. 46, no. 3, pp. 933-946, May 2000. 